\title{
A first simulation of a model aquatic canopy at high Cauchy number
}

B. Löhrer

Institute of Fluid Mechanics, TU Dresden, Germany

D. Doppler

Laboratoire de Mécanique des Fluides et d'Acoustique, CNRS - UMR 5509/Université de Lyon, École Centrale de Lyon/Université Lyon 1/INSA Lyon, Écully, France

S. Puijalon

UMR 5023 LEHNA, Université Lyon 1, CNRS, ENTPE, Villeurbanne Cedex, France

\author{
N. Rivière \\ Laboratoire de Mécanique des Fluides et d'Acoustique, CNRS - UMR 5509/Université de Lyon, École \\ Centrale de Lyon/Université Lyon 1/INSA Lyon, Écully, France
}

\section{J.J.S. Jerome}

Laboratoire de Mécanique des Fluides et d'Acoustique, CNRS - UMR 5509/Université de Lyon, École Centrale de Lyon/Université Lyon 1/INSA Lyon, Écully, France

\section{J. Fröhlich}

Institute of Fluid Mechanics, TU Dresden, Germany

\begin{abstract}
Canopies formed by flexible aquatic plants constitute an important element in river hydraulics as they determine resistance, resilience towards erosion, transport of nutrients and pollutants, and provide the habitat of other species. Due to their flexibility they undergo reconfiguration and closely interact with the surrounding flow. This fluid-structure-coupling poses a considerable challenge to detailed numerical modelling. In the present contribution a recently developed own method is presented capable of numerically simulating such a situation. It is applied to a model configuration composed of very long flexible blades which corresponds to an experiment presently conducted in Lyon. A particularity of this situation, so far considered only rarely, is the length and high flexibility of the blades leading to a Cauchy number $C a$ around 25,000. The parameters are such that the monami phenomenon is observed featuring synchronized undulating motion of the blades. This very detailed simulation at high $\mathrm{Ca}$ is the first of its kind with very long, flat blades typical for aquatic plants. The contribution reports first results for the velocity field in terms of highly resolved instantaneous data and one-point statistics.
\end{abstract}

Keywords: canopy, reconfiguration, high Cauchy number, water channel, scale-resolving simulation 


\section{INTRODUCTION}

Canopies formed by aquatic plants appear abundantly in water bodies under the most diverse circumstances and play decisive roles in the respective ecosystems. Of particular relevance are canopies in flowing streams due to the mechanical interaction between the mean and instantaneous fluid velocity and the deformation of the plants undergoing substantial reconfiguration with impact on flow resistance, sediment retention, species transport, etc. (Nepf 2012). Reconfiguration depends on the flow velocity and the properties of the solid bodies. It is usually expressed as a function of the Cauchy number $C a$ which is the ratio of the characteristic dynamic pressure of the fluid to the forces created by the stiffness of a structure. Consequently, low Cauchy numbers yield small deformation, while high Cauchy numbers entail large deformation.

To investigate fundamental issues it is convenient to consider prototypical canopies made of blades with identical shape, usually rectangular, and equal size and stiffness, together with a regular arrangement. This insures repeatability of experiments or simulations. Experiments with such canopies have been reported in several studies, such as (Kouwen \& Unny 1973) or (Rominger \& Nepf 2014), to name but two.

Simulations of such a setting are much scarcer due to the difficulties in modeling. Statistical turbulence models including deformation have been employed (Dijkstra \& Uittenbogaard 2010) Turbulence-resolving approaches, however, are very rare and more recent due to their high numerical cost. While such computations have been done in considerable number for rigid blades or beams, still demanding large computing times, the representation of fluid-structure coupling in the case of flexible blades which, in addition, also collide, constitutes a further challenge for the numericist.

In the literature few studies have been conducted with model canopies of low to moderate $C a$, (Boothroyd et al. 2016; Tschisgale et al. 2017). But to the knowledge of the authors only (Marjoribanks et al. 2017) so far numerically simulated canopies with very high $C a$, i. e. very flexible elements, in this case cylinders in a very regular staggered arrangement and with a relatively low resolution of 5 grid points per diameter.

Experiments for high- $C a$ canopies are difficult since the individual elements move substantially, making measurements complicated, and since, additionally, this motion obstructs the optical access to the interior of the canopy, whatever their arrangement.

The present contribution reports on a simulation of a high- $\mathrm{Ca}$ canopy presently mounted for experimental investigation at LMFA, Lyon. This canopy is made of long, flat ribbons of plastic with a density slightly smaller than that of water. It was devised to mimic patches of aquatic plants that are frequently formed by plants in streams (Cornacchia et al. 2018; (Licci et al. 2019). The linear strap-like shape of the blades is common among aquatic plant species (e. g. Sparganium emersum, Vallisneria spiralis) and is considered as an adaptation to flowing water (Sand-Jensen 2003; Albayrak et al. 2012), which makes the experimental configuration used in this study relevant to investigate interactions between hydrodynamics and plants.

\section{NUMERICAL METHOD}

Simulations were performed using the in-house code PRIME developed at TU Dresden (Kempe \& Fröhlich 2012; (Tschisgale et al. 2018). With this method, the Navier-Stokes equations for incompressible flows are solved employing a second-order finite volume approach on a staggered Cartesian grid with constant step size in all directions. For the time integration a semi-implicit pressure-correction approach using a three-step, second-order Runge-Kutta method is applied. Energetic turbulent scales are resolved and sub-grid scale turbulence is accounted for via a Large Eddy Simulation (LES) approach, a subgrid-scale viscosity provided by the Smagorinsky model.

The flexible structures forming the canopy are represented by means of the geometrically exact Cosserat rod model (Antman 1995) suitable for rods undergoing large deflections. Their motion is computed by a finite-difference approach according to (Lang \& Arnold 2012). Collisions are handled by the model proposed in (Tschisgale et al. 2019).

Coupling between fluid and structures is established with a recently developed new variant of the Immersed-Boundary Method (IBM) imposing a no-slip condition on the surface of the rods. The present implementation extends the approach of Tschisgale et al. (2018) and is applicable for 
small and even vanishing thickness of the blades. Details of the entire method and validations are compiled in (Tschisgale \& Fröhlich, subm.).

\section{PHYSICAL SETUP}

A fully developed open-channel flow is investigated with fluid properties corresponding to those of water at a temperature of $293.15 \mathrm{~K}$ according to the IAPWS97 standard (see Tab. 1). A set of 132 flexible blades with the properties given in Table 2 are attached perpendicular to the bottom wall at the locations indicated in Figure 1 . This pattern consists of seven unique columns in $z$ direction repeated in such a way that the resulting pattern cannot be subdivided into equal parts. The resulting array of blades is staggered in crosswise direction to avoid channelization.

Table 1. Fluid properties.

\begin{tabular}{lcl}
\multicolumn{2}{l}{ Open water channel, } & \\
$H=$ & $156 \mathrm{~mm}$ & channel height \\
$L_{z}=$ & $\begin{array}{l}250 \mathrm{~mm} \\
\text { channel width }\end{array}$ \\
$\rho=$ & $998.2 \mathrm{~kg} \mathrm{~m}^{-3}$ & fluid density \\
$\nu=1.003 \cdot 10^{-6} \mathrm{~m}^{2} \mathrm{~s}^{-1}$ & kin. viscosity \\
$U_{\mathrm{b}}=$ & $0.129 \mathrm{~m} \mathrm{~s}^{-1}$ & bulk velocity \\
\hline
\end{tabular}

Table 2. Structure properties.

\begin{tabular}{ll}
\hline \multicolumn{2}{l}{ LDPE strips under gravity $g=9.81 \mathrm{~m} / \mathrm{s}^{2}}$, \\
$L=250 \mathrm{~mm}$ & blade length \\
$W=15 \mathrm{~mm}$ & blade width \\
$T=0.063 \mathrm{~mm}$ & blade thickness \\
$\rho_{\mathrm{s}}=920 \mathrm{~kg} \mathrm{~m}^{-3}$ & structure density \\
$E=250 \mathrm{MPa}$ & Young's modulus \\
$G=83 \mathrm{MPa}$ & Shear modulus
\end{tabular}

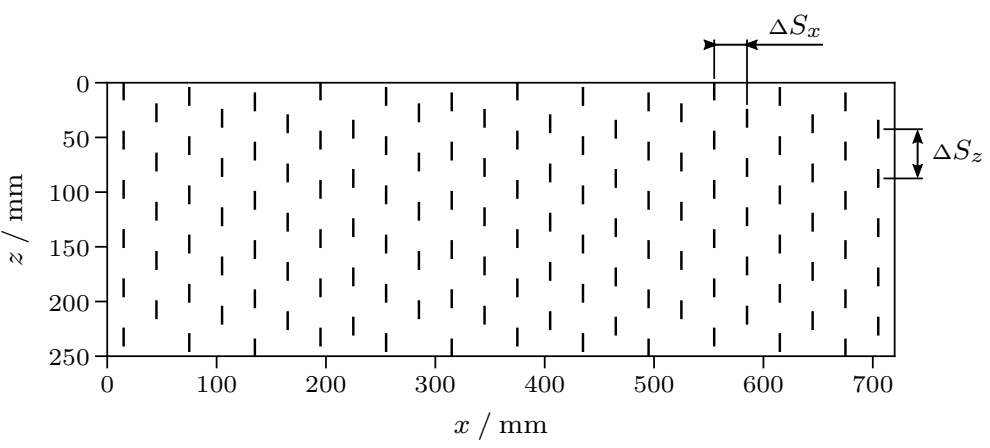

Figure 1. Pattern of locations for the fixation of flexible blades at the bottom wall, with $\Delta S_{x}=30 \mathrm{~mm}$ and $\Delta S_{z}=45 \mathrm{~mm}$.

Table 3. Dimensionless numbers.

\begin{tabular}{lrl}
\hline$C a=25,000$ & Cauchy number \\
$B=-145$ & Buoyancy number \\
$R e_{W}=1922$ & Reynolds number \\
$R e_{H}=20,064$ & Reynolds number \\
$\lambda \quad=0.38$ & Roughness density \\
\hline
\end{tabular}

To characterize the overall problem, the following dimensionless numbers are used,

$$
C a=\frac{\rho U_{\mathrm{b}}^{2} W L^{3}}{2 E I}, \quad B=\frac{\left(\rho_{\mathrm{s}}-\rho\right) g W T L^{3}}{E I}, \quad R e_{H}=\frac{U_{\mathrm{b}} H}{\nu}, \quad R e_{W}=\frac{U_{\mathrm{b}} W}{\nu},
$$

where $I=T^{3} W / 12$ is a second moment of area. The respective values for the present case are provided in Table 3. The huge Cauchy $C a$ and the relatively large value of $|B|$ reflect the enormous flexibility of the blades, i. e. both, nominal drag and lift force outweigh elastic forces. The roughness density $\lambda$ is defined as the ratio of the mean frontal area of a plant divided by the bed area it occupies. The latter quantity is approximated by $\Delta S_{x} \Delta S_{z}=13.5 \mathrm{~cm}^{2}$, the former 
requires knowledge of the mean reconfigured canopy height which is a results of the simulation. The computed value provided in Table 3 satisfies $\lambda C_{\mathrm{d}}>0.23$, indicative of a dense canopy (Poggi et al. 2004). In addition, with the reconfigured canopy height $L^{*} \approx 0.22 H$ the case is in the regime of shallow submergence, $H>L^{*}>H / 5$ where boundary layer turbulence is usually not developed and canopy scale turbulence dominates (Nepf 2012).

\section{NUMERICAL SETUP}

Values for several numerical parameters are provided in the Tables 4 5 . Periodic boundary conditions were imposed in $x$-direction, a rigid lid condition at the top wall, i. e. a flat geometry with free slip condition which is standard in simulations of this type (McSherry et al. 2017), and a no slip condition at the other three walls. The flow was driven by a spatially constant volume force adjusted in each time step to obtain the desired flow rate. This, as well, is standard for such a configuration.

While the Cosserat model accounts for bending, torsional, and shearing motion, the model assumption of constant cross section geometry prevents warping. In the present case of slender cross sections, however, warping is substantial. To remedy this issue, the effect of warping was modeled by reducing torsional and shearing stiffnesses by factors $k_{\mathrm{t}}$ and $k_{\mathrm{s}}$, respectively, with corresponding values computed as proposed by Freund \& Karakoç (2016).

Statistics were collected after a startup time $T_{\mathrm{st}} \approx 46 T_{\mathrm{b}}$, and during an averaging period $T_{\mathrm{av}} \approx 111 T_{\mathrm{b}}$, where $T_{\mathrm{b}}=H / U_{\mathrm{b}} \approx 1.21 \mathrm{~s}$. The time-averaged shapes of the individual blades were further averaged to obtain one mean shape. Its maximum elevation was taken as the mean reconfigured canopy height $L^{*}$.

Table 4. Num. settings of the fluid solver.

\begin{tabular}{lcl}
\hline$L_{x}=$ & $720 \mathrm{~mm}$ & domain length in $x$ dir. \\
$N_{x}=$ & 1152 & cells in $x$ direction \\
$N_{y}=$ & 250 & cells in $y$ direction \\
$N_{z}=$ & 400 & cells in $z$ direction \\
$C_{\mathrm{S}}=$ & 0.15 & Smagorinsky const. \\
$\Delta t=7 \cdot 10^{-4} \mathrm{~S}$ & time step size \\
\hline
\end{tabular}

Table 5. Numerical settings of the structure solver.

$\begin{array}{lrl}k_{\mathrm{s}}= & 0.833 & \text { shear corr. factors } \\ k_{\mathrm{t}}=7.14 \cdot 10^{-5} & \text { corr. factor for torsional warping } \\ N_{\mathrm{e}}= & 108 & \text { number of elements }\end{array}$

\section{RESULTS}

Figures 2 and 3 provide visualizations of the instantaneous flow for the same instant in time with the streamwise velocity component $u$ plotted in multiple slices. The visualizations give the impression of a substantial level of turbulence ranging from small structures at the scale of the blade width to very large ones. The latter is evident from the slightly meandering streamwise streaks of large velocity with a low-velocity region in-between (Fig. 3).

The blades undergo large deformations and exhibit undulations reaching up to about half the channel height with convex bending when seen from above. They also exhibit lateral motion, related to sideward inclination of their cross-section.

The profile of mean streamwise velocity $\langle u\rangle_{x, z, t}$ provided in Figure $4 \mid a$ contains two inflection points in the canopy region which is typical for dense canopies (Poggi et al. 2004). Interestingly, though, the upper one is located at $y \approx 0.08 H \approx 0.36 L^{*}$, with $L^{*} \approx 0.22 H$ the mean reconfigured canopy height, whereas in flows at lower $C a$ it is expected to be above the averaged canopy, e. g. observed by Marjoribanks et al. (2017). Another significant feature of the $\langle u\rangle_{x, z, t}$ profile is its maximum clearly below the surface known to be due to secondary flow structures in open-channel flows (Nezu \& Nakagawa 1993). Such a secondary flow, indeed, is seen in preliminary graphs. Streamwise fluctuations are particularly intense near the canopy top, $y=L^{*} \approx 0.22 \mathrm{H}$ as can be seen from the profile of $\left\langle u^{\prime} u^{\prime}\right\rangle_{x, z, t}$ in Figure 4t. Although not yet quantitatively ascertained, this is expected to be due to large vertical shifts of the horizontally aligned structures separating low and high momentum flow. The maxima of $-\left\langle u^{\prime} v^{\prime}\right\rangle_{x, z, t}$ and $\left\langle v^{\prime} v^{\prime}\right\rangle_{x, z, t}$ coincide at a position 
clearly above the average canopy top. In contrast, Ghisalberti \& Nepf (2002) found these maxima to occur near $y=L^{*}$ in case of lower values of $C a$, with that of $-\left\langle u^{\prime} v^{\prime}\right\rangle_{x, z, t}$ slightly below $y=L^{*}$ and the other just above it. This difference is assumed to be attributed to the rather prone mean geometry of the blades. Consequently, a blade at mean reconfiguration would be lowered by a sweep only slightly, soon being hindered by neighboring plants downstream, while an ejection has the potential to simultaneously lift an entire patch of several structures.

In videos, at rare instances, the blades are observed to interpenetrate and stick together for a moment. This is a technical defect in the implementation of the collision model. Its impact on the statistics is assumed negligible, but is subject to further investigation.

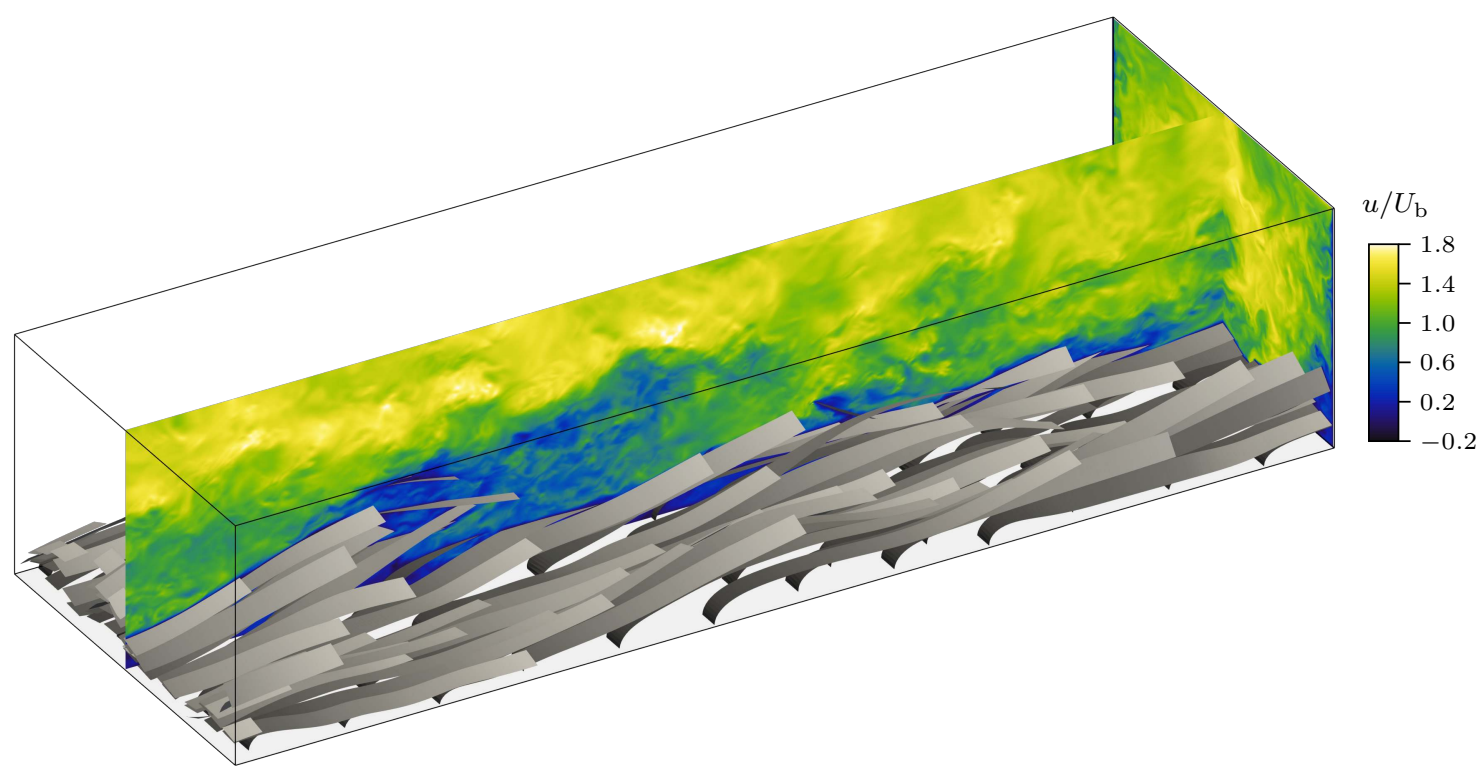

Figure 2. Instantaneous snapshot with the streamwise velocity component plotted in vertical planes. The structures are colored with a gradient (fading from root to tip) to render the individual structures more distinguishable.

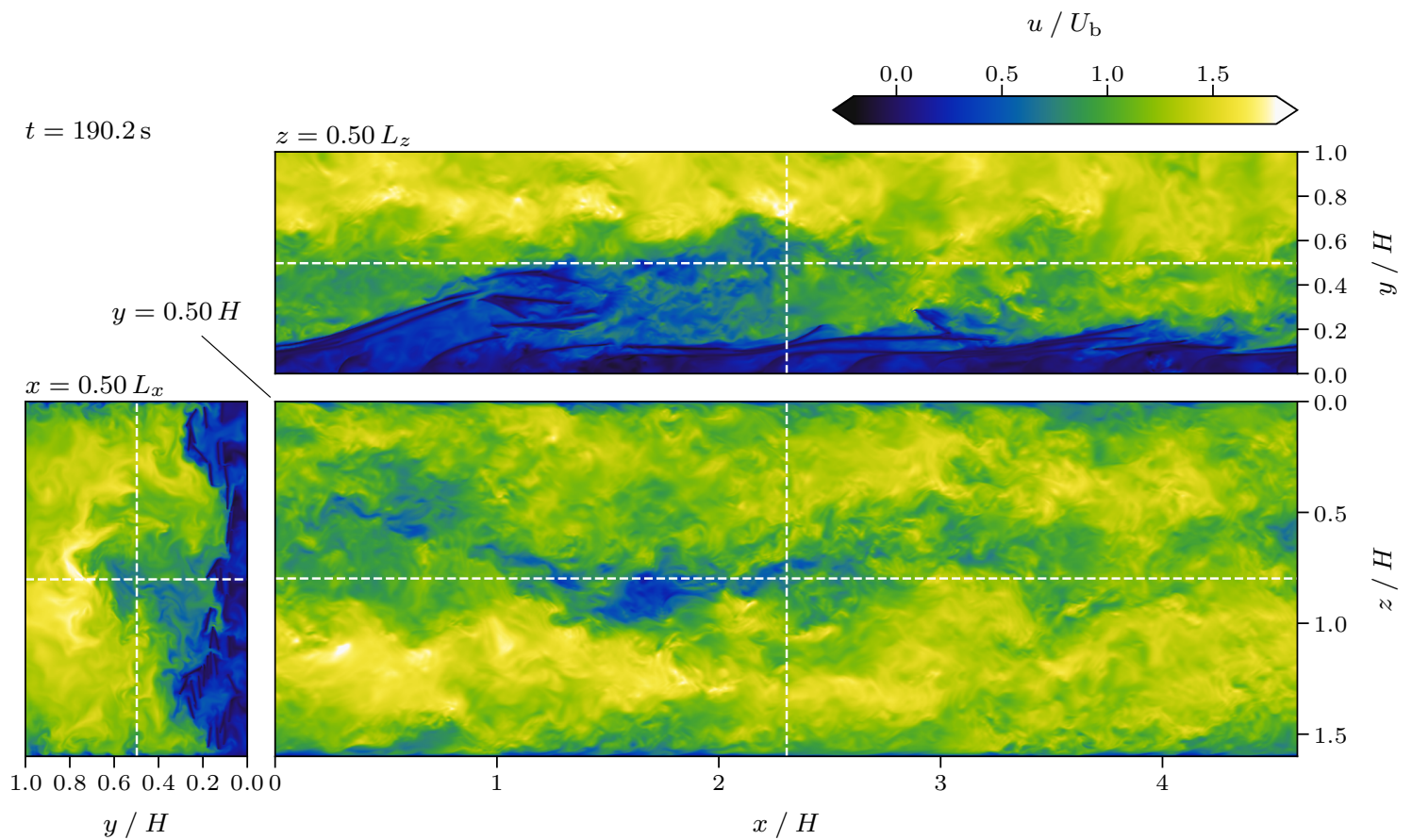

Figure 3. Instantaneous streamwise velocity for the same instant in time as in Figure 2 plotted in the slices indicated by the white dashed lines. 

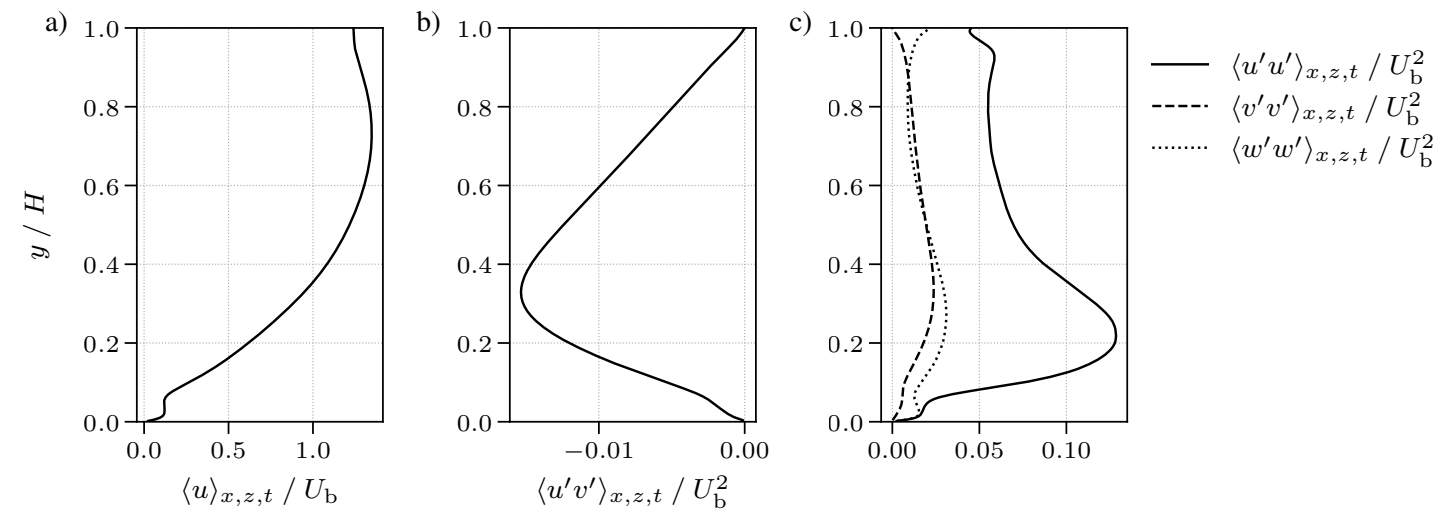

Figure 4. Mean streamwise velocity and Reynolds stress components, all obtained by averaging in $x, z$ (from wall to wall) and in time.

\section{CONCLUSIONS}

In this study a configuration was investigated that features a streamwise homogeneous canopy of long artificial blades with high flexibility mimicking a class of patches of aquatic plants. So far, experimental data are not yet available for this configuration, but the measurements are prepared at the time of writing. This is why the simulation features lateral walls, as these are present in the experiment.

The statistical results constitute a first step towards the analysis of this flow, indicating qualitative differences from more rigid canopies. This will be continued by means of two-point correlations and spectra. Future work will also be concerned with the motion of the blades and the corresponding statistics, as well as conditional averaging to investigate features of the coupling between blades and turbulent flow.

Acknowledgements This work was funded through the DFG-ANR project ESCaFlex. Computation time was provided by ZIH Dresden.

\section{REFERENCES}

Albayrak, I., Nikora, V., Miler, O., \& O’Hare, M. 2012. Flow-plant interactions at a leaf scale: Effects of leaf shape, serration, roughness and flexural rigidity. Aquatic Sciences 74(2), 267286.

Antman, S.S. 1995. Nonlinear Problems of Elasticity, Volume 107 of Applied Mathematical Sciences. New York, NY: Springer New York.

Boothroyd, R.J., Hardy, R.J., Warburton, J., \& Marjoribanks, T. I. 2016. The importance of accurately representing submerged vegetation morphology in the numerical prediction of complex river flow. Earth Surface Processes and Landforms 41(4), 567-576.

Cornacchia, L., van de Koppel, J., van der Wal, D., Wharton, G., Puijalon, S., \& Bouma, T.J. 2018. Landscapes of facilitation: How self-organized patchiness of aquatic macrophytes promotes diversity in streams. Ecology 99(4), 832-847.

Dijkstra, J.T. \& Uittenbogaard, R.E. 2010. Modeling the interaction between flow and highly flexible aquatic vegetation. Water Resources Research 46, W12547.

Freund, J. \& Karakoç, A. 2016. Warping displacement of Timoshenko beam model. International Journal of Solids and Structures 92-93, 9-16.

Ghisalberti, M. \& Nepf, H.M. 2002. Mixing layers and coherent structures in vegetated aquatic flows. Journal of Geophysical Research 107(C2).

Kempe, T. \& Fröhlich, J. 2012. An improved immersed boundary method with direct forcing for the simulation of particle laden flows. Journal of Computational Physics 231(9), 3663-3684.

Kouwen, N. \& Unny, T. 1973. Flexible roughness in open channels. Journal of the Hydraulics Division 99(hy5), 713-728. 
Lang, H. \& Arnold, M. 2012. Numerical aspects in the dynamic simulation of geometrically exact rods. Applied Numerical Mathematics 62(10), 1411-1427.

Licci, S., Nepf, H., Delolme, C., Marmonier, P., Bouma, T.J., \& Puijalon, S. 2019. The role of patch size in ecosystem engineering capacity: A case study of aquatic vegetation. Aquatic Sciences 81(3), 41.

Marjoribanks, T.I., Hardy, R.J., Lane, S.N., \& Parsons, D.R. 2017. Does the canopy mixing layer model apply to highly flexible aquatic vegetation? Insights from numerical modelling. Environmental Fluid Mechanics 17(2), 277-301.

McSherry, R.J., Chua, K.V., \& Stoesser, T. 2017. Large eddy simulation of free-surface flows. Journal of Hydrodynamics, Ser. B 29(1), 1-12.

Nepf, H.M. 2012. Flow and Transport in Regions with Aquatic Vegetation. Annual Review of Fluid Mechanics 44(1), 123-142.

Nezu, I. \& Nakagawa, H. 1993. Turbulence in Open-Channel Flows. IAHR-AIRH Monograph Series. Rotterdam: Balkema. OCLC: 832157663.

Poggi, D., Porporato, A., Ridolfi, L., Albertson, J.D., \& Katul, G.G. 2004. The effect of vegetation density on canopy sub-layer turbulence. Boundary-Layer Meteorology 111(3), 565-587.

Rominger, J.T. \& Nepf, H.M. 2014. Effects of blade flexural rigidity on drag force and mass transfer rates in model blades. Limnology and Oceanography 59(6), 2028-2041.

Sand-Jensen, K. 2003. Drag and reconfiguration of freshwater macrophytes. Freshwater Biology 48(2), 271-283.

Tschisgale, S. \& Fröhlich, J. submitted. An immersed boundary method for the fluid-structure interaction of slender flexible structures in viscous fluid.

Tschisgale, S., Kempe, T., \& Fröhlich, J. 2018. A general implicit direct forcing immersed boundary method for rigid particles. Computers \& Fluids 170, 285-298.

Tschisgale, S., Meller, R., \& Fröhlich, J. 2017. Simulation of the turbulent flow over an array of flexible blades. In 10th International Symposium on Turbulence and Shear Flow Phenomena (TSFP10), Chicago, USA.

Tschisgale, S., Thiry, L., \& Fröhlich, J. 2019. A constraint-based collision model for Cosserat rods. Archive of Applied Mechanics 89(2), 167-193. 